\title{
XMM-Newton survey of IGM: news for the modified entropy scaling
}

\author{
Alexis Finoguenov* \\ Max-Planck-Institut für extraterrestrische Physik, Giessenbachstraße, 85748 Garching, \\ Germany. alexis@mpe.mpg.de
}

\section{Stefano Borgani}

Dipartimento di Astronomia dell'Università di Trieste, via Tiepolo 11, I-34131 Trieste, Italy

INFN - National Institute for Nuclear Physics, Trieste, Italy. borgani@ts . astro. it

\section{David S. Davis}

Joint Center for Astrophysics, Physics Department, University of Maryland, Baltimore County, Baltimore, MD 21250

Laboratory for High Energy Astrophysics, NASA Goddard Space Flight Center, Code 661.0,

Greenbelt, MD 20771, USA. ddavis@milkyway.gsfc.nasa.gov

\section{John S. Mulchaey}

Observatories of the Carnegie Institution of Washington, 813 Santa Barbara Street, Pasadena,

CA 91101, USA. mulchaeyeociw.edu

\section{John P.F. Osmond, and Trevor J. Ponman}

School of Physics and Astronomy, University of Birmingham, Edgbaston, Birmingham B15 2TT,

UK. tjp@star.sr.bham.ac.uk

\section{Marc Zimer}

Max-Planck-Institut für extraterrestrische Physik, Giessenbachstraße, 85748 Garching,

Germany. zimer@mpe.mpg. de

Results of XMM-Newton survey of 40 galaxy groups are presented with accent on the global properties of the gas, such as entropy and pressure. We confirm the modified entropy scaling relation of Ponman, Sanderson \& Finoguenov (2003) at central parts of the groups $\left(0.1 r_{500}\right)$. At the same time, we show distinct differences between the virialized groups and emission originated from halos of early-type galaxies. The slopes of the entropy profiles of the groups are on average flatter than 1.1 law, typical of the clusters. We present the results of numerical simulations using the P-GADGET-II code, which are the first simulations capable to reproduce these newly observed trends.

Baryons in Dark Matter Halos

5-9 October 2004

Novigrad, Croatia

\footnotetext{
${ }^{*}$ Speaker.
} 


\section{Introduction}

Groups of galaxies constitute the most common galaxy association, containing as much as 50$70 \%$ of all galaxies in the nearby universe (Geller \& Huchra 1983) and provide a link between the massive virialized systems such as clusters of galaxies and the field. For example, Finoguenov et al. (2003b) found that chemical enrichment in groups is very close to the prediction of the Salpeter Initial Mass Function, known to characterize the star-formation in the field. It was also suggested by Kodama et al. (2001) that properties of galaxies in clusters are largely defined by their previous group environment. Thus, studies of groups open prospects to understanding galaxy formation, presently one of the most challenging astrophysical issues.

Although systems we study are defined by galaxy concentration, the presence of X-ray emission introduces further refinement in the definition and indicates that some of these systems are also virialized objects (Ostriker et al. 1995). Groups with a high fraction of early-type galaxies are more likely to exhibit detectable X-ray emission (Mulchaey et al. 1996). However, at low X-ray luminosities $\left(\sim 10^{41} \mathrm{ergs} \mathrm{s}^{-1}\right)$ the contribution from emission of galaxies becomes non-negligible. Some early-type galaxies have rather compact X-ray emission (Matsushita 2001; Finoguenov \& Miniati 2004), while others have been shown to lack a massive dark matter halo (Romanowsky et al. 2003). As bona fide collapsed halos exhibit some well-defined X-ray scaling relations, one can use these relationships to shed light on the large-scale dark matter distributions in these systems.

Comparative studies of the scaling relations in clusters of galaxies reveal strong deviations of the observed relations from predictions based on self-similar collapse (e.g. Ponman et. al. 1999; Finoguenov et al. 2001). These deviations are thought to be best characterized by the injection of energy (preheating) into the gas before clusters collapse (Kaiser 1991; Evrard \& Henry 1991). This entropy 'floor' causes the simple self-similar scaling laws (Kaiser 1986) to predict a shallower $L_{X}-T$ relationship. However, analysis of a large compilation of entropy profiles on groups and clusters of galaxies is inconsistent with this picture, requiring at $r_{500}$ much larger entropy levels than was thought before (Finoguenov et al. 2002) and modifying the concept of the entropy floor to the entropy ramp at $0.1 r_{200}$ (Ponman et al. 2003). Reproduction of these results both analytically and numerically, strongly supports the scenario of Dos Santos \& Dore (2002), where an initial adiabatic state of the infalling gas is further modified by the accretion shock (Ponman et al. 2003; Voit et al. 2003; Voit \& Ponman 2003). As a supporting evidence to the latter, Ponman et al. (2003) noticed a self-similarity in the entropy profiles, once scaled to $T^{0.65}$. Some XMM-Newton observations are consistent with this result (Pratt \& Arnaud 2003; Finoguenov et al. 2004). A major change introduced by these studies is that groups of galaxies can again be viewed as a scaled-down versions of clusters, yet the scaling itself is modified. Other evidence for the departure of groups from the trends seen in clusters has been recently been refuted by Osmond \& Ponman (2004).

In addition to preheating, internal heating by supernovae and AGN may also impact the observed scaling relations. Recent results from Chandra demonstrate that AGN heating is likely very important in both the centers of clusters (McNamara et al. 2001) and early-type galaxies (Finoguenov \& Jones 2001). However, very little work has been done to explore the role of AGN heating on the intragroup medium.

To better understand the physics of the intragroup medium, we (Finoguenov et al. 2004) have embarked on an XMM-Newton archival study of nearby galaxy groups. The large field of view 
of XMM-Newton, combined with its good resolution and large collecting area make it an ideal instrument to study the hot gas in nearby groups. We also perform a study of how structure in the observed systems may affect their appearance on the scaling relations for entropy. We adopt a Hubble constant of $70 \mathrm{~km} \mathrm{~s}^{-1} \mathrm{Mpc}^{-1}, \Omega_{M}=1-\Omega_{\Lambda}=0.3$, and present redshifts corrected for the local motion.

\section{Sample Selection}

At the moment there is no large purely $\mathrm{X}$-ray selected sample of groups of galaxies. For example, only a few objects, Fornax cluster, MKW4, NGC 4636, NGC 1550 and NGC 5044 are present in the HIFLUGS, a complete all-sky sample of brightest groups and clusters of galaxies (Reiprich \& Böhringer 2002). Most present-day samples of groups are based on the X-ray followup of optical surveys (e.g. Mahdavi et al. 2000; Mulchaey et al. 2003).

For this study we have selected the groups in Mulchaey et al. (2003) with publicly available XMM-Newton observations. Most of the groups in the Mulchaey et al. (2003) sample were found by cross-correlating the ROSAT observation log with the positions of optically-selected groups. Their final group list contains 109 systems.
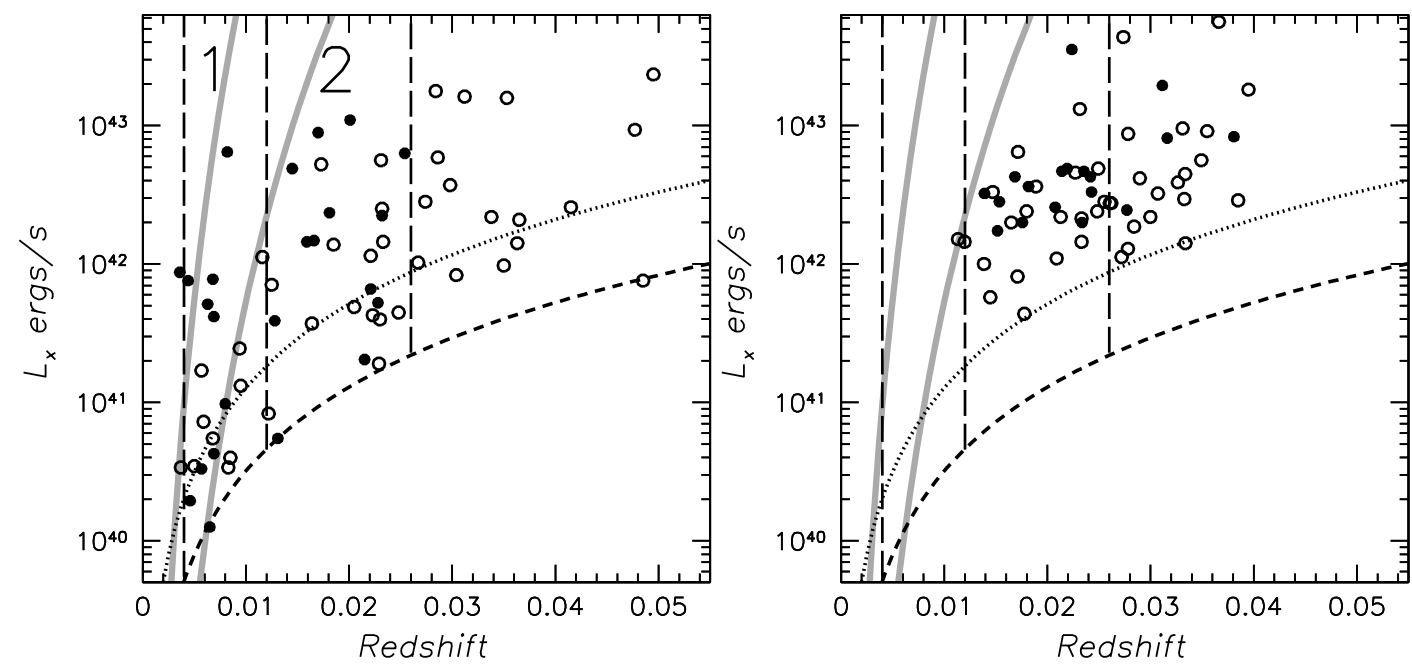

Figure 1: Left panel. Observed characteristics of the 2DXGS. The circles correspond to groups listed in Mulchaey et al. (2003). Filled circles denote objects observed by XMM-Newton. The change in the luminosity limit corresponding to the same X-ray flux in correspondence to Mulchaey and Mahdavi surveys is shown with short-dashed and dotted lines, respectively. The vertical long-dashed lines define the two subsamples in 2DXGS and are labeled 1 and 2. The XMM field-of-view will enclose $1 / 4$ of the virial radius for the groups to the right of low redshift grey line and $1 / 2$ of the virial radius for the higher redshift grey line. The quoted luminosities are bolometric assuming $h_{100}$ (Mulchaey et al. 2003) and are measured out to the radius of X-ray detection. Right panel. The same as the left panel, but for the Mahdavi et al. (2000) group sample. The luminosities are given for $0.1-2.4 \mathrm{keV}$ band, $h_{100}$ and aperture of $500 \mathrm{kpc}$ (Mahdavi et al. 2000). For systems in common with the Mulchaey et al. (2003) sample, these luminosities are lower by $0.1-0.2$ dex, which is accounted for by the bolometric correction.

The properties of groups from the Mulchaey et al. (2003) sample are shown in Fig.1. The flux limit corresponds to $2.6 \times 10^{-13} \mathrm{ergs} \mathrm{s}^{-1} \mathrm{~cm}^{-2}$ at a $3 \sigma$ detection level, which is a factor of 4 lower than the flux limit in the Mahdavi et al. (2000) sample, a factor of 10 lower than in the REFLEX/NORAS surveys and a factor of 100 lower compared to HIFLUGS (these latter surveys 
are all based on the RASS data). The distribution of systems in the plot is quite homogeneous, as are the targets observed by XMM-Newton (which covers $50 \%$ of groups at redshifts lower than 0.025; independent off the volume and luminosity cut). By construction, the Mulchaey et al. (2003) group list covers a fraction of a percent of the sky for serendipitous systems, yet it includes a larger fraction of bright objects, that were selected as primary targets for ROSAT pointings, e.g. 3 of the 4 HIFLUGS groups are included.

\section{Intragroup or Individual Galaxy Emission?}

The XMM-Newton data allow us to compare the entropy and pressure behavior at radii exceeding $0.1 r_{500}$ for a large sample of groups for the first time. Based on the levels of entropy and pressure, we separate our sample onto four categories: 1) regular groups (NGC 5044, NGC 5846), 2) groups disturbed externally (NGC 4636), 3) groups disturbed internally (IC 1459, NGC 2300 and NGC 4261), and 4) systems dominated by individual galaxies (NGC 3665, NGC 5322, NGC 3923, NGC 4168). The first three categories are introduced in attempt to explain the observed variation in the entropy level among the groups, while the last category is composed of objects that may not have a virialized group-size halo. As the division is entirely based on the entropy level, a system of low X-ray luminosity is considered a group if its temperature is proportionally low, while a different origin of the emission is suggested if the temperature is high. With the current data in hand these two categories are not overlapping in the entropy at radii exceeding $0.3 r_{500}$. Normally, the scaling for entropy starts to work already at $0.1 r_{500}$ and to cope with the observational evidence we introduce so-called internally disturbed systems.

The regular groups are selected to exhibit an entropy similar to bona fide groups and cool clusters from previous ASCA (Ponman et al. 2003) and current XMM-Newton studies (Pratt \& Arnaud 2003; Finoguenov et al. 2005; Mahdavi et al. 2004). While interaction is also seen in such groups, it is unable of changing the baseline level for the entropy and pressure outside the central region. In this section we sample the separation between different categories of groups, and later compare the hydrodynamical simulations to the observations of the thermodymanical state of the gas at outskirts of the groups in the higher-redshift counterpart of this study.

NGC 4636 is the only group in our sample whose X-ray properties appear to be shaped by external forces. In particular, the NGC 4636 system appears to be interacting with the Virgo cluster: NGC 4636 is moving to the south with the plume representing a tail of emission left behind from this motion.

The third category represents objects that have been significantly disturbed internally. The internal disturbances are so important in these systems that the hot gas returns to a more normal distribution only at very large radii $\left(0.2-0.4 r_{500}\right)$. In the cases of IC 1459 and NGC 4261, powerful central radio galaxies are likely responsible for disturbing the gas. For NGC 4261 this is quite apparent as the radio emission fills in the "holes" in the X-ray emission, as has now been found for many clusters observed with Chandra (Bîrzan et al. 2004). In the case of the NGC 2300 group, the interaction between the central galaxy and NGC 2276 is probably responsible for the bow shaped structure seen in the pressure map. This interaction is also appears responsible for inducing the star-burst in the NGC 2276 galaxy (Gruendl et al. 1993). 

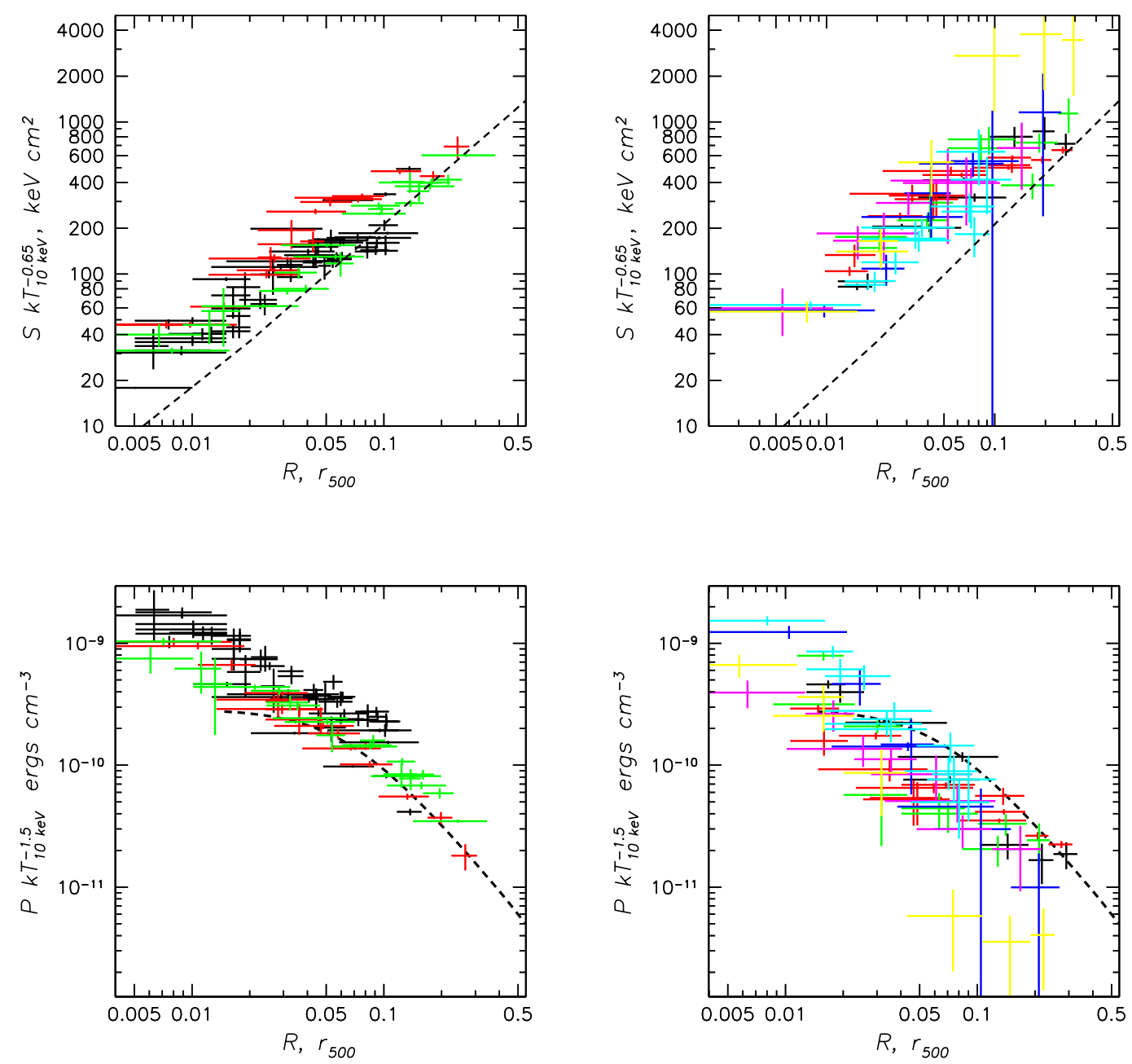

Figure 2: Scaled entropy (top) and pressure (bottom) profiles for the sample. Left panel: NGC 4636 (black), NGC 5846 (red), NGC 5044 (green). Right panel: NGC 2300 (black), NGC 4261 (red), IC 1459 (green), NGC 3665 (blue), NGC 4168 (magenta), NGC 3923 (cyan), NGC 5322 (yellow). The universal entropy scaling relation of Ponman et al. (2003) is shown with a dashed-dotted line on both entropy panels. A typical pressure profile is shown as a dashed line on both pressure panels.

The entropy levels of the final category of objects is much higher than the other groups in our sample and the ASCA sample of Ponman et al. (2003). The low pressure and high entropy at all radii in these systems can not be explained by infall of the gas associated with group formation. Thus, it is probably more proper to think of the X-ray emission in these systems as originating in the ISM of the galaxies and not as the intragroup medium. Certainly (looking at the normalization of the pressure profiles) most of the systems in question have masses that make $10 \%$ of the NGC 5044, which puts them into $10^{12} \mathrm{M}_{\odot}$ range, more typical of massive elliptical galaxies. The existence of such objects suggests the mere presence of X-ray emission in a system can be misleading. In particular, the presence of X-ray emission in a group does not always mean the system is a virialized object and the nature of these groups is still not clear. To complete the global properties of this category of groups, we note that the element abundance observed at X-rays is very low, that (under 
assumption of the robustness of optical metalicity indicators) contradicts to the suggestion of the origin of the gas from stellar mass loss. Additional insights to the origin of this weak component has been provided in higher redshift part of this survey, that included HCG92 (Finoguenov et al. 2005), a smoking gun of HI-to-X-ray conversion (Trinchieri et al. 2003). That study makes a conclusion that the faint X-ray components stems from a galaxy merger. It is relevant to point out that NGC 5322 has a counter-rotating core, which is most certainly a result of a recent merger. The high entropy level of the observed component requires cooling times longer than Hubble time to remove it (e.g. Voit et al. 2003), so we expect the faint X-ray halo to be a prominent feature, and a fossil record of merger history of early-type galaxies. Our arguments could be extended to include the faint (yet existing) X-ray appearance of post-merger galaxies, reported in Read \& Ponman (1998). On the other hand, the spiral dominated group studied here does not reveal the signatures of extended X-ray emission and thus we can rule out the scenario where such a component is a mere addition of elusive extended X-ray haloes of spiral galaxies.

\section{Reproduction of IGM thermodynamical properties in simulations.}
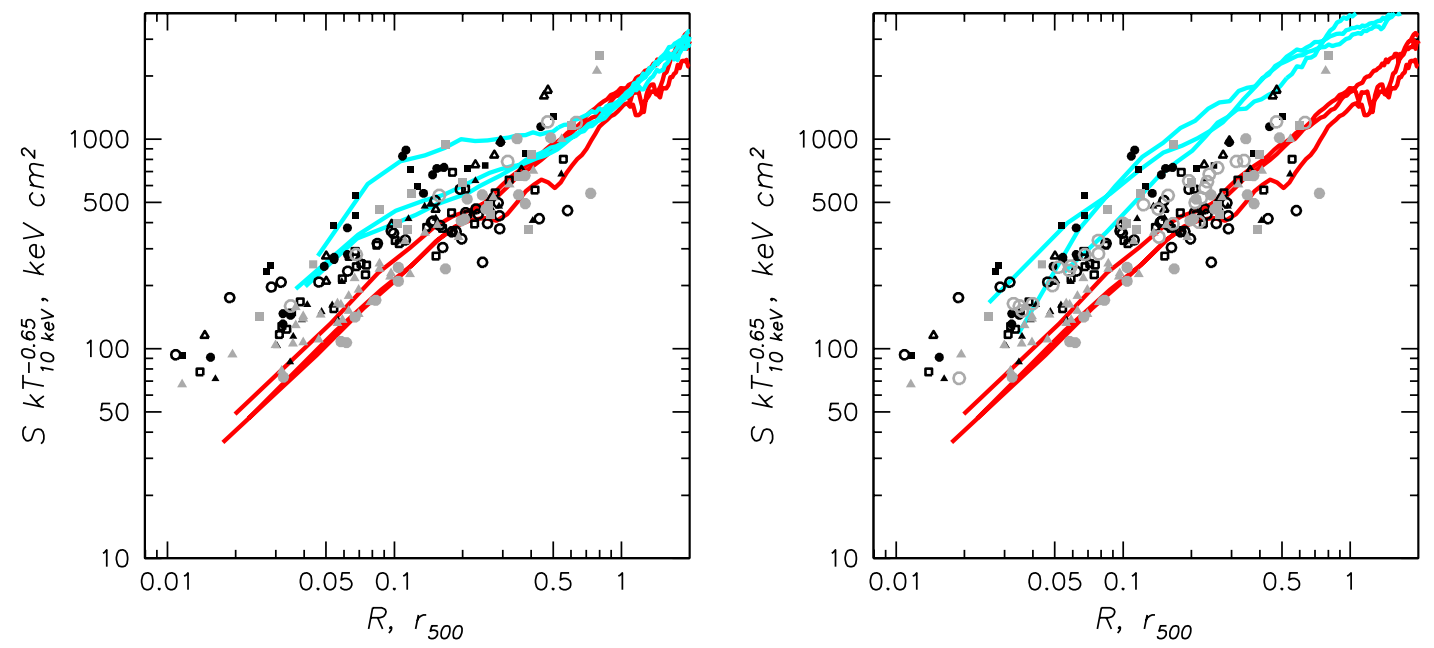

Figure 3: Scaled entropy from the $0.012<z<0.024$ sample of groups. The points corresponding to the same system are denoted by similar symbols. Red lines on both panels correspond to the results of the standard Hernquist $\&$ Springel (2003) wind model prescription. Several lines of the same color are present and indicate variation of the virial mass of the group. Blue lines present the results of investigation of the parameters space in the feedback models. On the left, stronger wind models are shown $(v=720 \mathrm{~km} / \mathrm{sec})$, on the right we set an entropy floor of $100 \mathrm{keV} \mathrm{cm}^{2}$ at $z=3$. While the energy budget of the two runs is similar, they differ in their prediction at outskirts of the groups. Comparison with the data suggests that the gas observed in outskirts of the groups has been able to cool down by the present epoch.

Our simulation were carried out using GADGET-2, a new version of the parallel TreeSPH code GADGET (Springel, Yoshida \& White 2001). It uses an entropy-conserving formulation of SPH (Springel \& Hernquist 2002), and includes radiative cooling heating by a uniform evolving UV background, a sub-resolution description of star formation from a multiphase interstellar medium, and the effect of galactic winds powered by SN energy feedback (Springel \& Hernquist 2003).

The simulated structures are the same presented by Tornatore et al. (2003, see also Finoguenov et al. 2003a) and we refer to that paper for further details. They are one moderately rich cluster with $M_{v i r} \simeq 2.6 \times 10^{14} M_{\odot}$, and three groups-sized objects having virial mass in the range $1.6 \times$ 
$10^{13} \lesssim M_{\text {vir }} / h^{-1} M_{\odot} \lesssim 4.2 \times 10^{13} M_{\odot}$. The corresponding halos have been selected from a DM-only simulation of a $\Lambda$ CDM model with $\Omega_{m}=0.3, h=0.7, \sigma_{8}=0.8$ and $f_{\text {bar }}=0.13$ for the baryon fraction, within a box of size $70 h^{-1} \mathrm{Mpc}$. As such, they encompass the mass range characteristic of moderately rich clusters to groups, where the effect of non-gravitational gas heating is expected to be more important. Further details are described in Borgani et al. (2005).

As a test to simulations, we have chosen to reproduce the behavior of the entropy in the sample of groups at $0.02<z<0.04$, the high-z part of the Mulchaey groups, described above. The comparison shown in Fig.3 suggests that the gas observed in outskirts of the groups has been able to cool down by the present epoch. By varying the strength of the galactic wind in the model of Hernquist \& Springel (2003) from 240 to $720 \mathrm{~km} / \mathrm{sec}$ we were able to encompass the scatter in the observed data.

\section{Acknowledgments}

The paper is based on observations obtained with XMM-Newton, an ESA science mission with instruments and contributions directly funded by ESA Member States and the USA (NASA). The XMM-Newton project is supported by the Bundesministerium für Bildung und Forschung/Deutsches Zentrum für Luft- und Raumfahrt (BMFT/DLR), the Max-Planck Society and the HeidenhainStiftung, and also by PPARC, CEA, CNES, and ASI. AF acknowledges support from BMBF/DLR under grant 50 OR 0207, MPG and a partial support from NASA grant NNG04GF686. The simulations have been realized with CPU time allocated at the "Centro Interuniversitario del Nord-Est per il Calcolo Elettronico" (CINECA, Bologna), thanks to grants from INAF and from the University of Trieste. This work has been partially supported by INFN (Grant PD-51).

\section{References}

[1] Borgani, S., Finoguenov, A., Kay, S.T., Ponman, T.J., Springel, V., Tozzi, P., Voit, G.M. 2005, MNRAS, subm.

[2] Bîrzan, L., Rafferty, D. A., McNamara, B. R., Wise, M. W., \& Nulsen, P. E. J. 2004, ApJ, 607, 800

[3] Dos Santos, S. \& Doré, O. 2002, A\&A, 383, 450

[4] Finoguenov, A., Reiprich, T., Böhringer, H. 2001, A\&A, 368, 749

[5] Finoguenov, A. \& Jones, C. 2001, ApJL, 547, L107

[6] Finoguenov, A., Jones, C., Böhringer, H., \& Ponman, T. J. 2002, ApJ, 578, 74

[7] Finoguenov A., Borgani S., Tornatore L., Böhringer H., 2003a, A\&A, 398, L35

[8] Finoguenov, A., Burkert, A., Böhringer, H. 2003b, ApJ, 594, 136

[9] Finoguenov, A., Davis, D.S., Zimer, M., Mulchaey, J.S. 2004, ApJ, subm.

[10] Finoguenov, A., Miniati, F. 2004, A\&A, 418, L21

[11] Finoguenov, A., Ponman, T.J., Osmond, J.P.F., Zimer, M. 2005, MNRAS, subm.

[12] Geller, M. J., \& Huchra, J. P. 1983, ApJS, 52, 61

[13] Gruendl, R. A., Vogel, S. N., Davis, D. S., Mulchaey, J. S. 1993, ApJL, 413, L81 
[14] Hernquist, L., \& Springel, V. 2003, MNRAS, 341, 1253

[15] Kaiser, N. 1986, MNRAS, 222, 323

[16] Kaiser, N. 1991, ApJ, 383, 104

[17] Kodama, T., Smail, I., Nakata, F., Okamura, S., \& Bower, R. G. 2001, ApJL, 562, L9

[18] Mahdavi, A., Böhringer, H., Geller, M. J., \& Ramella, M. 2000, ApJ, 534, 114

[19] Mahdavi, A., Finoguenov, A., Böhringer, H., Geller, M. J., Henry, J. P. 2004, ApJ, accepted

[20] Matsushita, K. 2001, ApJ, 547, 693

[21] McNamara, B. R., Wise, M. W., Nulsen, P. E. J., David, L. P., Carilli, C. L. 2001, ApJL, 562, L149

[22] Mulchaey, J. S., Davis, D. S., Mushotzky, R. F., Burstein, D. 1996, ApJ, 456, 80

[23] Osmond, J. P. F. \& Ponman, T. J. 2004, MNRAS, 350, 1511

[24] Ostriker, J. P., Lubin, L. M., Hernquist, L. 1995, ApJ, 444, L61

[25] Ponman, T. J., Bourner, P. D. J., Ebeling, H., Böhringer, H. 1996, MNRAS, 283, 690

[26] Ponman, T. J., Cannon, D. B., Navarro, J. F. 1999, Nature, 397, 135

[27] Ponman, T. J., Sanderson, A. J. R., Finoguenov, A. 2003, MNRAS, 343, 331

[28] Pratt, G. W., Arnaud, M. 2003, A\&A, 408, 1

[29] Read, A. M. \& Ponman, T. J. 1998, MNRAS, 297, 143

[30] Reiprich, T. H. \& Böhringer, H. 2002, ApJ, 567, 716

[31] Romanowsky, A. J., Douglas, N. G., Arnaboldi, M., et al. 2003, Science, 301, 1696

[32] Springel V., Yoshida N., White S.D.M., 2001, NewA, 6, 79

[33] Springel, V., \& Hernquist, L. 2002, MNRAS, 333, 649

[34] Springel, V., \& Hernquist, L. 2003, MNRAS, 339, 289

[35] Tornatore L., Borgani S., Springel V., Matteucci F., Menci N., Murante G., 2003, MNRAS, 342, 1025

[36] Trinchieri, G., Sulentic, J., Breitschwerdt, D., \& Pietsch, W. 2003, A\&A, 401, 173

[37] Voit, G. M., Balogh, M. L., Bower, R. G., Lacey, C. G., \& Bryan, G. L. 2003, ApJ, 593, 272

[38] Voit, G. M. \& Ponman, T. J. 2003, ApJL, 594, L75 